\title{
A BAG3 chaperone complex maintains cardiomyocyte function during proteotoxic stress
}

Luke M. Judge, ${ }^{1,2}$ Juan A. Perez-Bermejo, ${ }^{2,3}$ Annie Truong, ${ }^{2}$ Alexandre J.S. Ribeiro, ${ }^{2,4}$ Jennie C. Yoo, ${ }^{2}$ Christina L. Jensen, ${ }^{2}$ Mohammad A. Mandegar, ${ }^{2}$ Nathaniel Huebsch, ${ }^{2}$ Robyn M. Kaake, ${ }^{2}$ Po-Lin So, ${ }^{2}$ Deepak Srivastava, ${ }^{1,2}$ Beth L. Pruitt, ${ }^{4,5}$ Nevan J. Krogan, ${ }^{2,3}$ and Bruce R. Conklin ${ }^{2,6}$

'Department of Pediatrics, UCSF, San Francisco, California, USA. ${ }^{2}$ Gladstone Institutes, San Francisco, California, USA. ${ }^{3}$ Department of Cellular and Molecular Pharmacology, UCSF, San Francisco, California, USA. ${ }^{4}$ Stanford Cardiovascular Institute and Mechanical Engineering Department, and ${ }^{5}$ Bioengineering and Molecular and Cellular Physiology Departments, Stanford University, Stanford, California, USA. ${ }^{6}$ Department of Medicine, UCSF, San Francisco, California, USA.

Molecular chaperones regulate quality control in the human proteome, pathways that have been implicated in many diseases, including heart failure. Mutations in the BAC3 gene, which encodes a co-chaperone protein, have been associated with heart failure due to both inherited and sporadic dilated cardiomyopathy. Familial BAC3 mutations are autosomal dominant and frequently cause truncation of the coding sequence, suggesting a heterozygous loss-of-function mechanism. However, heterozygous knockout of the murine BAC3 gene did not cause a detectable phenotype. To model BAC3 cardiomyopathy in a human system, we generated an isogenic series of human induced pluripotent stem cells (iPSCs) with loss-of-function mutations in BAC3. Heterozygous BAC3 mutations reduced protein expression, disrupted myofibril structure, and compromised contractile function in iPSC-derived cardiomyocytes (iPS-CMs). BAC3-deficient iPS-CMs were particularly sensitive to further myofibril disruption and contractile dysfunction upon exposure to proteasome inhibitors known to cause cardiotoxicity. We performed affinity tagging of the endogenous BAC3 protein and mass spectrometry proteomics to further define the cardioprotective chaperone complex that BAC3 coordinates in the human heart. Our results establish a model for evaluating protein quality control pathways in human cardiomyocytes and their potential as therapeutic targets and susceptibility factors for cardiac drug toxicity.

equity in Tenaya, and Tenaya provides research support for heart failure related research. B.R. Conklin also serves on the advisory board or as a consultant to Arc Bio, Cellogy Inc., Scientist.com, Allen Institute for Cell Science (Seattle), The Exploratorium (San Francisco), Innovative Genomics Institute (Berkeley), Chinese University of Hong Kong. D. Srivastava also holds an equity in Tenaya. M.A. Mandegar is currently an employee of Tenaya Therapeutics. L.M. Judge and B.R. Conklin are authors on a pending patent application (International Publication Number W02017/027466), "Method of determining cellular protein homeostasis."

Submitted: April 18, 2017

Accepted: June 13, 2017

Published: July 20, 2017

Reference information: JCI Insight. 2017;2(14):e94623. https://doi.org/10.1172/jci. insight.94623.

\section{Introduction}

Cardiomyocytes must maintain constant contractile function throughout a human lifetime. As a result, the cells undergo continuous mechanical and oxidative stress, which leads to protein damage and misfolding that can impair contractile function and lead to formation and aggregation of toxic peptides (1). Additionally, the heart has minimal regenerative capacity, so cardiomyocytes must compensate for this proteotoxic stress by strictly regulating new protein synthesis and degrading damaged protein components (2). To this end, cardiac tissue maintains high levels of constitutive and tissue-specific chaperones in which rare mutations can cause severe disease $(3,4)$.

Protein quality control pathways are increasingly recognized for their importance in both inherited and sporadic cardiac disease, and as potential therapeutic targets (5). The co-chaperone BAG3 is particularly interesting, because genetic evidence suggests that variants of BAG3 can be both pathologic and protective. For example, the rare P209L missense mutation causes severe childhood-onset myofibrillar myopathy, a lethal disease that affects both skeletal and cardiac muscle, possibly through a toxic gain of function in the BAG3 protein $(4,6,7)$. Additionally, a variety of heterozygous mutations in BAG3, many of which are nonsense or frameshift mutations consistent with loss of function, cause autosomal dominant familial dilated cardiomyopathy (8-11). BAG3 has also been associated with acquired forms of cardiomyopathy, such as stress (Takotsubo) cardiomyopathy (12-14). Alternatively, a common coding polymorphism in BAG3 was associated with a significantly lower odds ratio for developing idiopathic dilated cardiomyopathy, suggesting that this polymorphism could be protective (9). In animal models, BAG3 mutations or deficiency generally result 
in myopathy phenotypes $(6,8,15)$. However, in mice, knockout of the BAG3 gene produced inconsistent findings: one group reported a primary myopathy phenotype and the other reported systemic pathology without a clear muscle phenotype $(15,16)$. Interestingly, neither group detected any phenotype in heterozygous null mice. Because BAG3-related cardiac disease in humans is most frequently associated with heterozygous lossof-function mutations, mice may not be an ideal model for the disease.

BAG3 is a stress-response gene induced by heat shock factor 1 (17). Although BAG3 is widely expressed in many tissues, it is most highly expressed in skeletal and cardiac muscle, where it localizes with the sarcomeric Z-disk (15). As a co-chaperone, the BAG3 protein modulates ATP turnover and the protein folding activity of HSC70 (constitutive) and HSP70 (induced) $(18,19)$. It also physically and functionally interacts with a separate family of chaperones, the small heat shock proteins (HSPB genes), and acts as a scaffold that links the functions of HSP70 and HSPB families (20-22). In human cells, the BAG3-HSP70-HSPB complex regulates degradation of ubiquitinated proteins via the proteasome and autophagy pathways (2326). However, these experimental studies used overexpression and/or transient knockdown in immortalized cell lines with uncertain physiologic relevance. Given the cardiac-specific pathology caused by human BAG3 mutations and the unique expression pattern of the protein in the target tissue, disease modeling will be most informative if performed in cardiomyocytes. Furthermore, to avoid spurious interactions produced by less precise methods, manipulation of the endogenous gene is the preferred method. To evaluate the role of BAG3 in the physiological and pathological functions of human cardiomyocytes, we generated an isogenic series of cardiomyocytes derived from genetically engineered human induced pluripotent stem cells (iPSCs). In this way, we aimed to more accurately recapitulate aspects of human cardiomyopathy and mechanisms that protect against cardiac stress.

\section{Results}

We detected BAG3 protein in the cytoplasm of iPSCs, which increased approximately 10-fold in iPSCderived cardiomyocytes (iPS-CMs), where it was enriched at the sarcomeric Z-disk and the perinuclear region (Supplemental Figure 1; supplemental material available online with this article; https://doi. org/10.1172/jci.insight.94623DS1). We first studied the effects of BAG3 deficiency in cardiomyocytes by generating loss-of-function mutations via engineering the endogenous BAG3 locus (27). The strategy was designed to mimic effects of early nonsense mutations such as R90X and R123X that were reported in dilated cardiomyopathy patients (8). To maximize the insights we might gain into molecular mechanisms and minimize the chances of common off-target editing, we used multiple genome-editing tools to create mutations at 2 different sites in the BAG3 gene (Figure 1). We isolated both heterozygous and homozygous clones with loss-of-function mutations using transcription activator-like effector nucleases (TALENs) and clustered regularly interspaced short palindromic repeat (CRISPR)/Cas9-based strategies targeting 2 different parts of the second exon (Figure 1 and Supplemental Figure 2). We verified the genotype of each engineered mutant line by sequencing. All genetically modified iPSC lines retained pluripotent growth and morphology, normal karyotype, expressed pluripotency markers, and could efficiently differentiate into cardiomyocytes (Supplemental Figures 3 and 4).

As predicted, BAG3 loss-of-function mutations decreased BAG3 protein expression (Figure 1 and Supplemental Figure 1). Mutant cardiomyocytes were maintained for more than 30 days of differentiation without displaying obvious abnormalities in cell viability, morphology, or beating. This result is consistent with the murine BAG3-knockout model that exhibits normal cardiac morphology at birth (15). Based on human clinical reports and animal studies, we hypothesized that BAG3-mutant cells would develop disrupted myofilament structure over time, particularly at the sarcomeric Z-disk $(4,6,15)$. To examine myofilament structure, we replated cardiomyocytes onto glass coverslips and cultured them for variable intervals of time before fixing and staining them for the sarcomeric Z-disk protein $\alpha$-actinin (ACTN2). Cardiomyocytes continued to beat after being plated on glass coverslips, but after 7 or more days we observed a dramatic disruption of the Z-disk structure in $B A G 3^{-/}$cardiomyocytes (Figure 1). To quantify this phenotype, we blindly scored the extent of sarcomeric disarray in individual fixed cells on a 5-point scale (Supplemental Figure 5) (28). We observed an increase in the proportion of BAG3-mutant cells with significantly disordered myofilaments compared with WT cells, and $B A G 3^{-1-}$ cells displayed a trend toward more severe phenotype than $B A G 3^{+/-}$cells (Figure 1 and Supplemental Figure 5). These results were consistent between lines generated by the different genome-targeting strategies, indicating that the phenotype was caused by the loss of BAG3 expression and not off-target effects. 

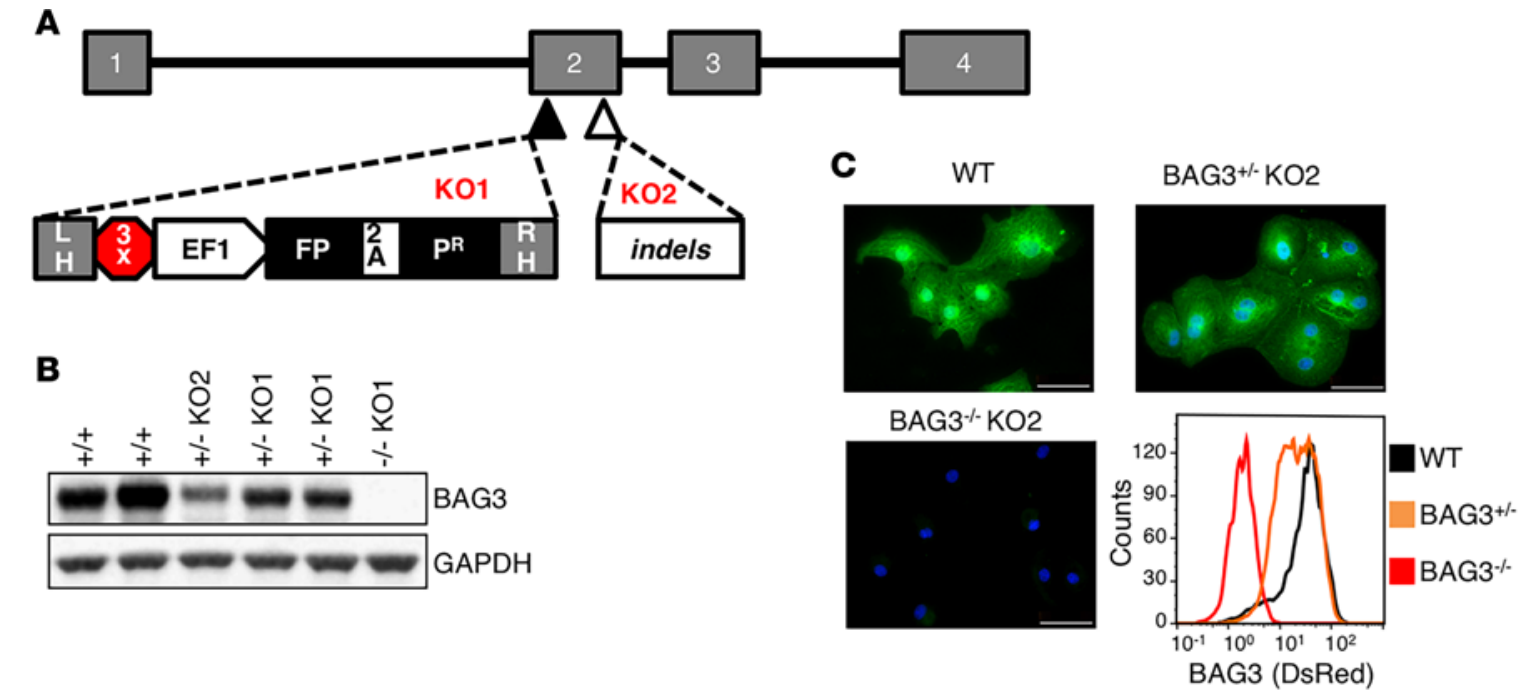

D
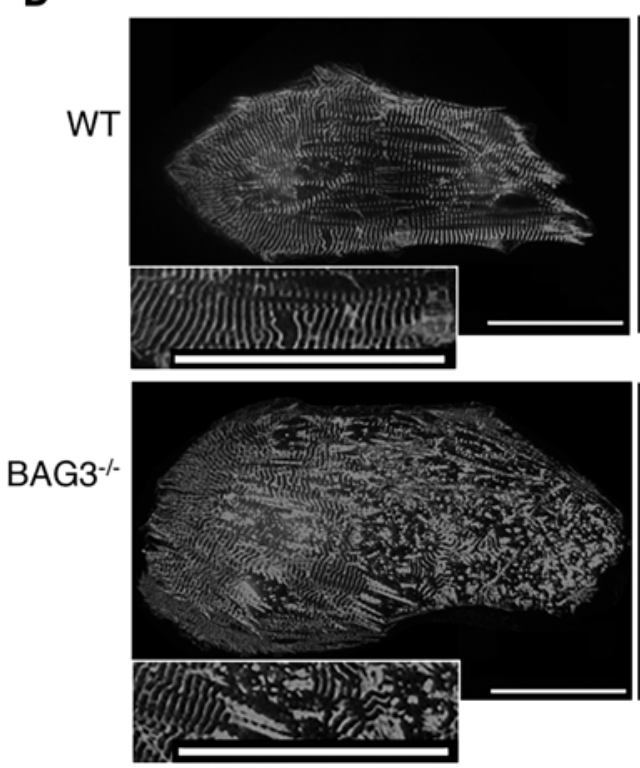
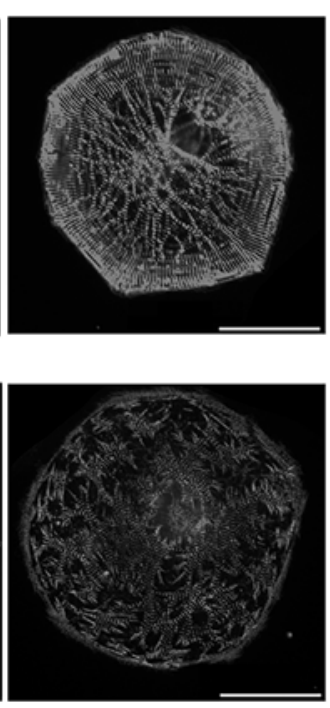

$\mathbf{E}$

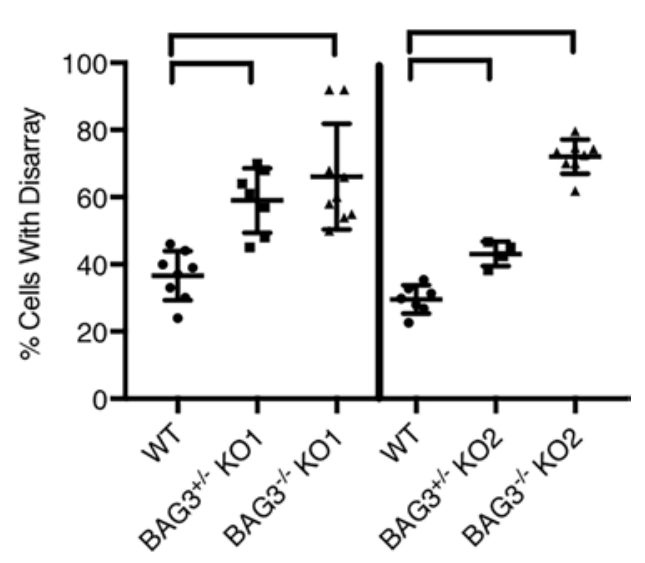

Figure 1. Genome engineering an isogenic series of BAG3 mutations leads to sarcomeric disarray in human induced pluripotent stem cell-derived cardiomyocytes (iPS-CMs). (A) Schematic of the BAC3 gene with 4 exons in the predominant coding isoform. Because an alternatively spliced isoform excludes the first exon, the second exon was targeted for knockout. BAC3-knockout lines were generated using TALEN-induced (black triangle) targeted integration of the knockout vector in exon 2 (KO1). The vector included flanking left and right homology arms (LH and RH, respectively) with a terminator sequence in 3 reading frames followed by the $\mathrm{mCherry}$ fluorescent protein (FP) and a puromycin selection cassette (P') driven by the EF1 $\alpha$ promoter. Transient expression of Cas9 with a guide RNA targeted downstream in exon 2 (open triangle) induced small insertions/deletions (indels) by nonhomologous end joining, resulting in frameshift and nonsense mutations (KO2). (B) Western blot for BAC3 protein in iPS-CMs. (C) Immunofluorescent staining and flow cytometry with

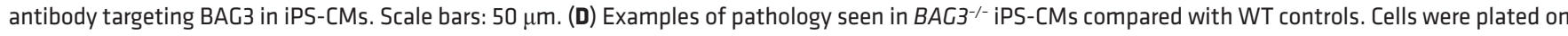
glass coverslips, fixed, and stained with antibody against ACTN2 to label Z-disks. Scale bars: $50 \mu \mathrm{m}$. (E) Quantification of sarcomeric disarray from blinded scoring on a 5-point scale, with disarray defined as the percentage of cells scored as class 3-5. Individual replicates are plotted with mean and SD. Brackets indicate significant differences $(P<0.001)$ by 1-way ANOVA with Bonferroni's test for multiple comparisons. For K01 analysis, 7-9 independent cultures per line from 3 separate differentiations were scored. For KO2 analysis, 4-8 independent cultures per line from 2 separate differentiations were scored.

Cardiomyocytes cultured on standard tissue culture surfaces frequently display a different morphologic shape and myofibril arrangement than that seen in normal tissue. Furthermore, culture on glass surfaces, which are extremely stiff, exposes the cells to an artificial mechanical environment. To evaluate cardiomyocyte contractile function under more physiologically relevant conditions, we cultured cardiomyocytes on micropatterned surfaces with constrained geometry and on polyacrylamide substrates of different stiffness (29). We then measured contractile function under those varying mechanical conditions. Cardiomyocytes were seeded onto rectangular patterns with a 7:1 aspect ratio to promote mature sarcomeric organization 
A

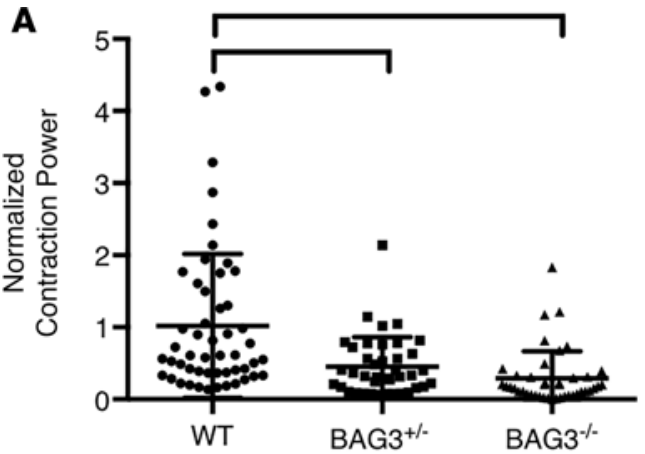

B

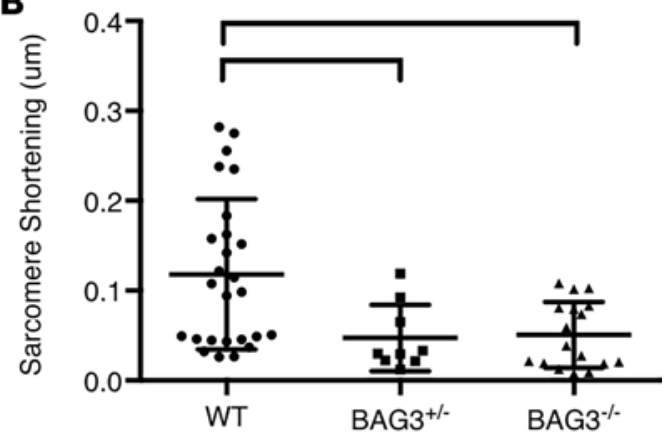

C

WT

BAG3 $\%$
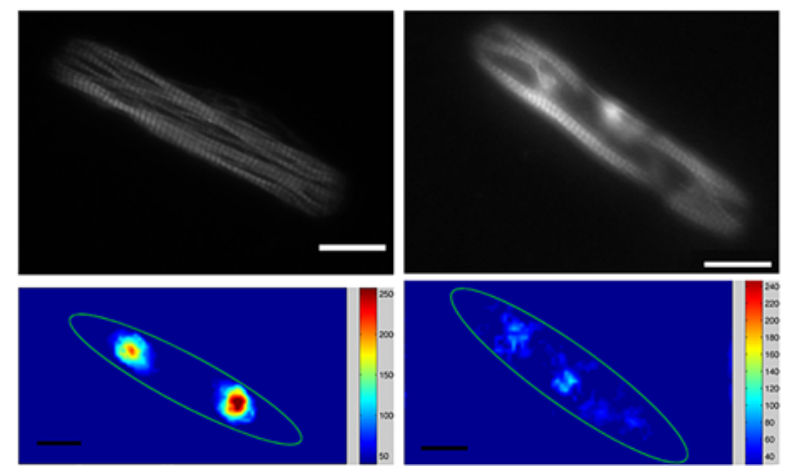

Figure 2. BAG3 mutations produce contractile deficits in iPS-CMs cultured on micropatterned substrates. Day $>30$ purified induced pluripotent stem cell-derived cardiomyocytes (iPS-CMs) were cultured on micropatterned polyacrylamide hydrogel substrates with a mechanical stiffness of $10 \mathrm{kPa}$. BAC3 KO1 mutant lines were used. (A) Contraction power was calculated from the measured force and contraction velocity determined by traction force microscopy from the movement of fluorescent beads in the substrate. Results were normalized to WT and individual replicates plotted with mean and SD. Brackets indicate significant differences $(P<0.001)$ by 1-way ANOVA with Bonferroni's test for multiple comparisons. (B) Sarcomere shortening was measured in LifeAct-labeled myofibrils. Results were normalized to WT and individual replicates plotted with mean and SD. Brackets indicate significant differences $(P<0.05)$ by 1-way ANOVA with Bonferroni's test for multiple comparisons. Measurements were obtained from 3 independent device cultures, prepared from 2 separate differentiation batches. For force measurements, 40-51 total cells were analyzed per line. For sarcomere shortening, 9-26 total cells were analyzed per line. (C) Representative images of patterned LifeAct-labeled cells, and associated heatmaps for surface traction stress (scale in Pa) are shown. Scale bars: $20 \mu \mathrm{m}$.

and function, with substrate stiffness mimicking physiologic $(10 \mathrm{kPa})$ or pathologically increased $(35 \mathrm{kPa})$ myocardial stiffness. Contraction power was calculated for individual cells by measuring the displacement of fluorescent beads embedded in the substrate as previously described (29). When cultured on substrates mimicking physiologic stiffness, $B A G 3^{+/-}$and $B A G 3^{-/-}$cardiomyocytes generated less contraction power than WT cardiomyocytes (Figure 2). To evaluate myofibril structure and measure sarcomere shortening, we also labeled patterned cells with LifeAct (29). With this approach, both $B A G 3^{+/-}$and $B A G 3^{-/-}$cardiomyocytes displayed defects in sarcomere shortening compared with WT cardiomyocytes (Figure 2). We repeated these experiments on substrates with $35 \mathrm{kPa}$ stiffness, which led to an overall reduction in contraction power and sarcomere shortening. However, there continued to be an additional deficit in these parameters in the $B A G 3^{--}$iPS-CMs compared with WT (Supplemental Figure 6). BAG3-mutant cardiomyocytes cultured on substrate with physiological stiffness also displayed disrupted myofibrils (Figure 2), supporting our initial findings. Thus, our data confirm that partial loss of BAG3 function compromises contractile performance in human cardiomyocytes, localized to disruption of myofibril structure and activity, supporting a dilated cardiomyopathy phenotype.

BAG3 participates in targeting ubiquitinated proteins for degradation via the proteasome or autophagy pathways $(26,30)$. Thus, we hypothesized that cardiomyocytes with defects in BAG3 co-chaperone activity would be further compromised by proteasome inhibition. Proteasome inhibitors are an important class of compounds recently developed for cancer therapy, although treatment can be complicated by cardiac toxicity, which has been observed in animals and humans $(31,32)$. To measure the effects of proteasome inhibitors on iPS-CM contractile motion, we used an automated video microscopy system (33). With this 


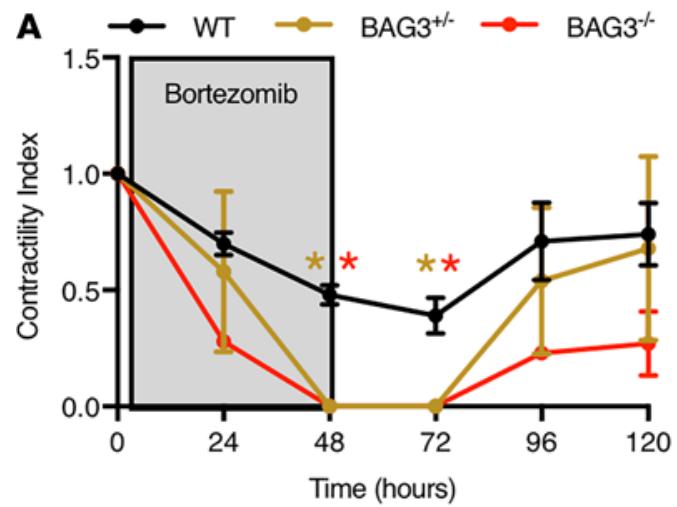

C
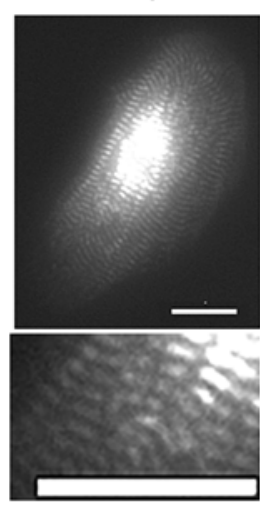

WT
$24 \mathrm{hr}$

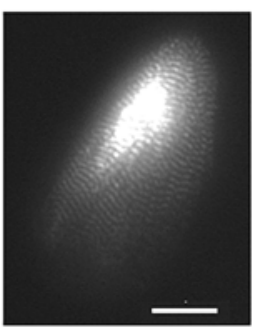

Bortezomib

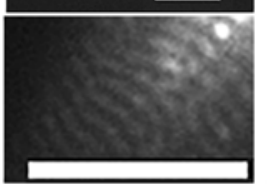

$48 \mathrm{hr}$
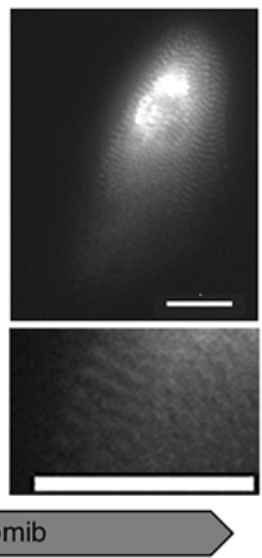

B $\multimap$ WT $\multimap$ BAG3 $^{+\%} \longrightarrow$ BAG3 $^{\%}$

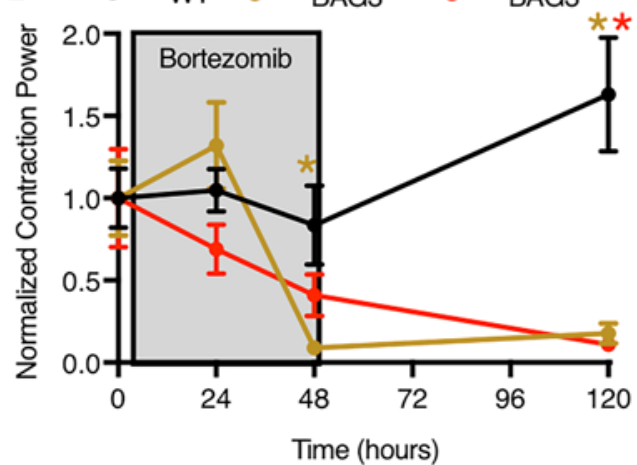

D

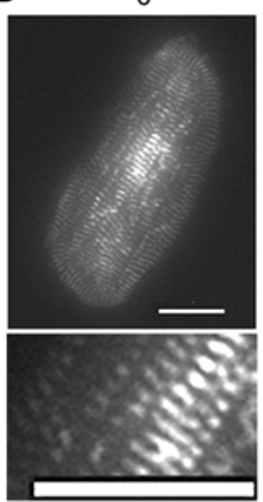

BAG3-/-
$24 \mathrm{hr}$
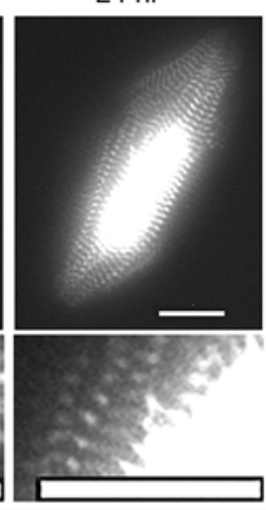

$48 \mathrm{hr}$

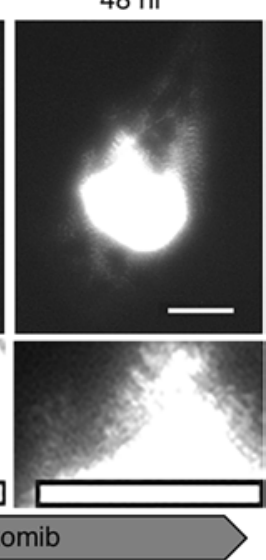

E DMSO $\rightleftarrows$ Bortezomib

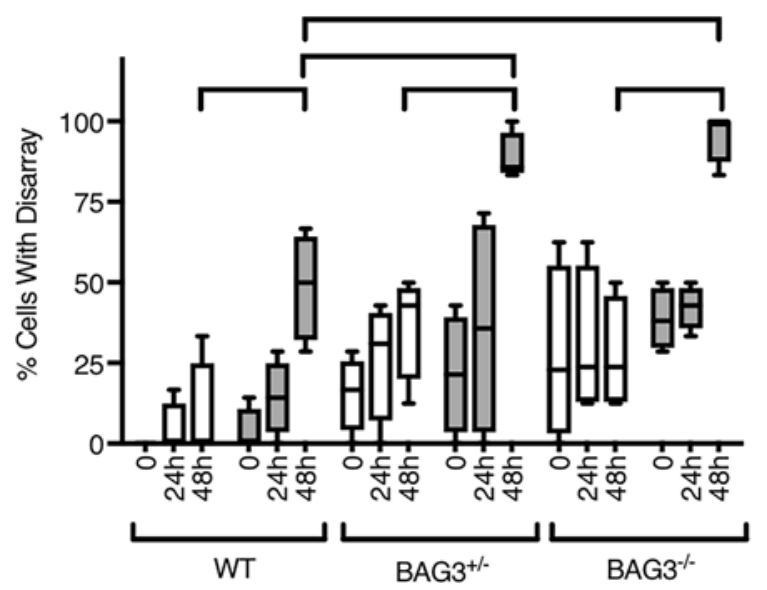

Figure 3. BAG3 is required to prevent severe cardiotoxicity from the proteasome inhibitor bortezomib. (A) Automated video microscopy system (Cellogy Pulse) was used to serially measure induced pluripotent stem cell-derived cardiomyocyte (iPS-CM) contractility before and every 24 hours after exposure to bortezomib $(0.1 \mu \mathrm{M})$. WT and BAC3-mutant (K01) iPS-CMs were exposed to drug for 48 hours and then allowed to recover in RPMI/B27 media for 3 days. Contractility index represents the contraction peak height at each time point normalized to the baseline value for each well. Mean and SEM are plotted from 4-8 independent replicates. ${ }^{*} P<0.05$ by 1-way ANOVA with Bonferroni's test for multiple comparisons. (B) WT and BAC3-mutant iPS-CMs cultured on micropatterned substrates mimicking physiologic stiffness (10 kPa) were exposed to bortezomib (0.1 $\mu \mathrm{M})$ for 48 hours and then allowed to recover in RPMI/B27 media for 3 days. Contraction power was measured at each time point and normalized to the baseline value for each population. Plotted are the mean and SEM of measurements from $9-24$ cells at each time point. ${ }^{*} P<0.05$ by 1 -way ANOVA with Bonferroni's test for multiple comparisons. (C and D) iPS-CMs were transfected with plasmid expressing fluorescent fusion protein ACTN2-mKate2 to label Z-disks. Individual cells were imaged at baseline and by time-lapse microscopy every 24 hours after treatment with DMSO $(0.01 \%)$ or bortezomib $(0.1 \mu \mathrm{M})$. Scale bars: $20 \mu \mathrm{m}$. (E) Individual cells were scored at each time point using the 5-point scoring system. The percentage of cells with disarray (class $3-5$ ) was calculated from 4 separate cultures for each condition and plotted as the mean, with boxes representing interquartile range and whiskers showing min-max. Brackets indicate significant differences $(P<0.05)$ by 2 -way ANOVA with Bonferroni's test for multiple comparisons. 

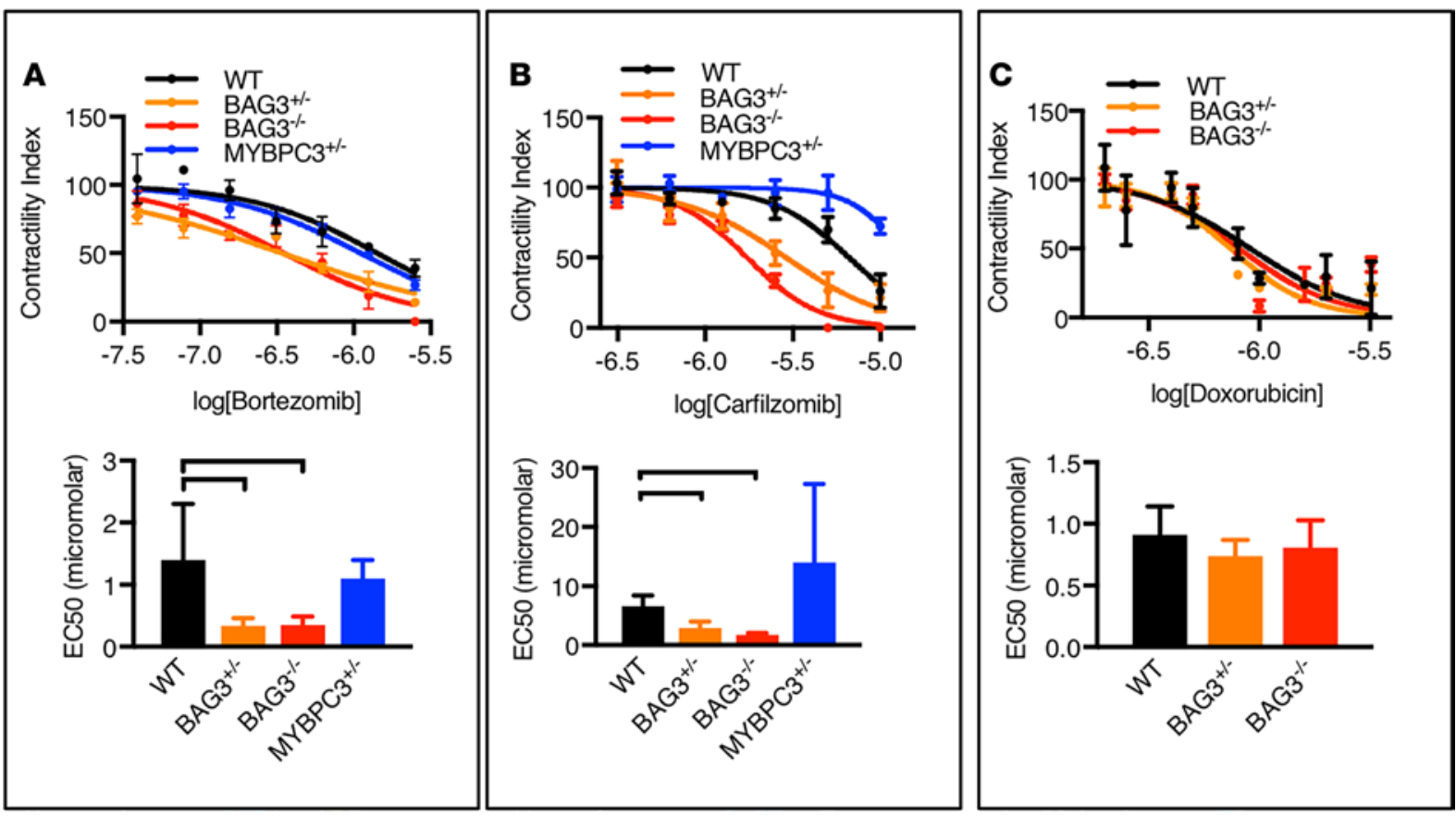

Figure 4. Genetic and pharmacologic specificity of the interaction between BAG3 and proteasome inhibitors. WT, BAC3-mutant (KO2), and MYBPC3mutant day >30 induced pluripotent stem cell-derived cardiomyocytes (iPS-CMs) were treated with (A) bortezomib, (B) carfilzomib, or (C) doxorubicin at the doses indicated. Contraction peak height was measured prior to and 48 hours after drug exposure, and results normalized to the baseline for each independent sample (contractility index). Mean and SEM are plotted for triplicate samples at each dose. Lower graphs represent the calculated $\mathrm{EC}_{50}$ and $95 \%$ confidence interval from each corresponding dose response curve using nonlinear regression analysis. Brackets indicate significant differences $(P<$ 0.05 ) by 1-way ANOVA with Bonferroni's test for multiple comparisons.

approach, we observed a dose-dependent decrease in iPS-CM contractility after a single exposure to 2 FDA-approved proteasome inhibitors, bortezomib and carfilzomib (Supplemental Figure 7). These effects occurred at concentrations well within the range of reported plasma concentrations after intravenous infusion in patients $(34,35)$. Contractility continued to decrease even after the proteasome inhibitors were removed, but it recovered after several days (except at the highest doses). Notably, bortezomib had more potent and longer-lasting effects on contractility than carfilzomib (Supplemental Figure 7).

With this same assay, we compared the effects of bortezomib on WT and BAG3-mutant cardiomyocytes. Exposure to $0.1 \mu \mathrm{M}$ bortezomib strikingly decreased contractility of BAG3-mutant iPS-CMs compared with WT controls (Figure 3). To confirm this result, we treated cardiomyocytes cultured on micropatterned substrates with physiologic stiffness with the same concentration and duration of bortezomib exposure, followed by serial measurements of contraction power. We found that, compared with WT cardiomyocytes, BAG3-mutant cardiomyocytes had a greater decrease in contraction power during exposure to bortezomib, and failed to recover contractile activity (Figure 3).

To determine whether the decrease in contractility caused by bortezomib was a result of disruption of myofilament structure, we expressed a fluorescently tagged ACTN2 to label Z-disks in live cells (36). Cells expressing the transgene were viable and continued to contract normally (Supplemental Video 1). We used time-lapse fluorescence microscopy to image cells with unambiguously labeled myofibrils at baseline, which revealed defects in myofilament structure within 48 hours of bortezomib treatment (Figure 3, C-E). We used the aforementioned scoring system to quantify the degree of myofibrillar disarray in these treated samples. As expected, $B A G 3^{+/-}$and $B A G 3^{-/-}$cardiomyocytes displayed more severely disrupted myofilament structure after treatment with bortezomib than WT cardiomyocytes (Figure 3E). These results support that BAG3 mutants failed to compensate for proteotoxic stress induced by proteasome impairment.

To ensure that the interaction between BAG3 mutations and proteasome inhibitors represented a specific biological mechanism rather than a general phenotype of myopathic disease, we performed several pharmacological experiments. For example, disparate genetic models of dilated cardiomyopathy in human iPS-CMs revealed a defective chronotropic response to adrenergic stimulation, along with an exacerbated 
A

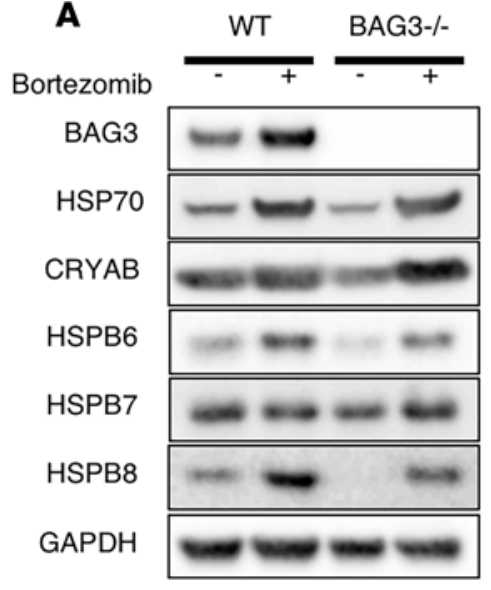

B

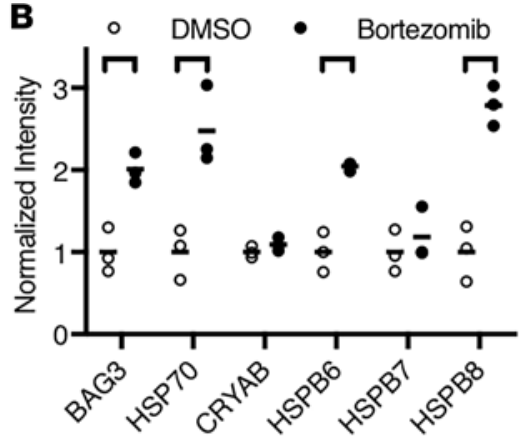

C

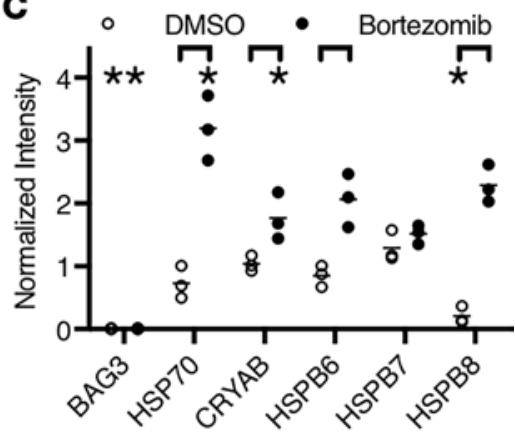

$\begin{array}{rccccccccc}\text { D Bort: } & - & - & - & - & - & - & + & + & + \\ \text { BafA1: } & - & - & - & + & + & + & + & + & + \\ \text { BAG3: } & +/+ & +/- & -/ & +/+ & +/- & -/ & +/+ & +/- & -/-\end{array}$

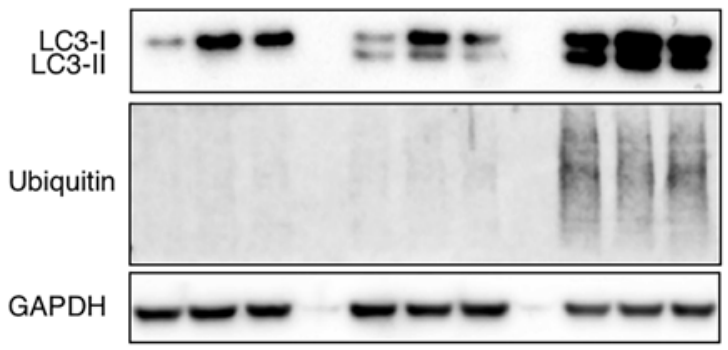

E

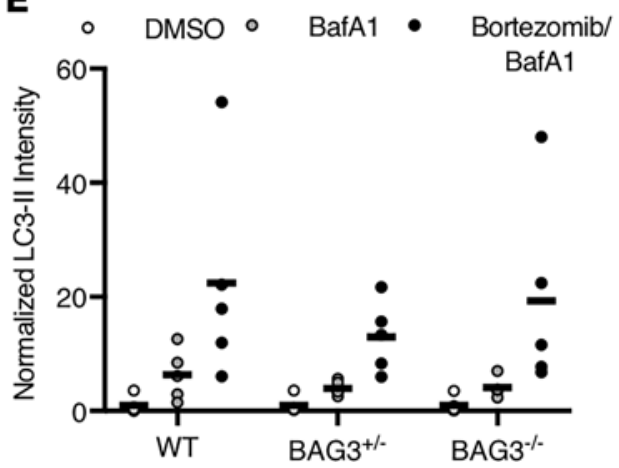

Figure 5. Bortezomib induces a cardiac chaperone stress-response and increases autophagy flux, the latter of which does not require BAG3. (A) Induced pluripotent stem cell-derived cardiomyocytes (iPS-CMs) were treated for 20 hours with vehicle control or $1 \mu \mathrm{M}$ bortezomib, followed by protein extraction and Western blot. A representative blot from 3 experiments is shown. (B and $\mathbf{C}$ ) Band intensities were quantified relative to GAPDH loading control and normalized to vehicle-treated WT control. WT cardiomyocytes treated with vehicle and bortezomib are compared in B, with BAG3 ${ }^{-/-}$cardiomyocytes treated with vehicle and bortezomib compared in C. Data represent individual values with horizontal lines indicating the mean. Brackets denote significant differences from corresponding vehicle-treated samples. ${ }^{*} P<0.05$ for $B A C 3^{-/-}$samples with significant difference from corresponding WT samples using 2-way ANOVA with Bonferroni's test for multiple comparisons. (D) iPS-CMs were treated with DMSO, 100 nM bafilomycin A1 (BafA1) for 6 hours, or $1 \mu$ M bortezomib for 14 hours followed by $100 \mathrm{nM}$ bafilomycin A1 for 6 hours. Total protein extracts were prepared and Western blot performed using antibody against LC3A/B. A representative blot from 5 experiments is shown. (E) LC3-II band intensities were quantified relative to GAPDH loading control and normalized to vehicle-treated sample for each cell line. Plotted are individual biological replicates, with horizontal lines indicating the mean. There was a statistically significant effect from drug treatment $(P<0.0001)$, but no significant difference between cell lines using 2-way ANOVA.

disease phenotype after repetitive dosing of norepinephrine (37, 38). However, BAG3-mutant lines displayed a positive chronotropic response to stimulation of both $\alpha$ - and $\beta$-adrenergic receptors by phenylephrine and isoproterenol, respectively (Supplemental Figure 8). Treatment with norepinephrine produced a similar chronotropic response in all cell lines over repeated dosing, without loss in contractility (Supplemental Figure 8). We also did not observe a significant increase in myofibrillar disarray after chronic adrenergic stimulation (data not shown).

Next, we performed dose-response assays to examine the effects of bortezomib and carfilzomib on contractility and viability using WT, BAG3-mutant, and MYBPC3-mutant iPS-CMs (Figure 4). Cardiac myosin-binding protein C (MYBPC3) is commonly mutated in cardiomyopathies (39), and based on affinity purification followed by mass spectrometry (AP-MS), it likely interacts with the BAG3 chaperone complex (see below). The $\mathrm{EC}_{50}$ for the inhibition of contractility by bortezomib and carfilzomib was significantly lower in both $B A G 3^{+/-}$and $B A G 3^{-/-}$cells compared with WT cells, but not $M Y B P C 3^{+/-}$cells (Figure 4). These effects on contractility were not simply due to cell death, since the effect of bortezomib on cell viability was only significantly different in the $B A G 3^{-1-}$ cells, and carfilzomib had minimal effects on viability at doses that suppressed contractility (Supplemental Figure 9). To further confirm specificity of drug toxicity, we repeated these assays with doxorubicin, a cardiotoxic chemotherapy agent. By 


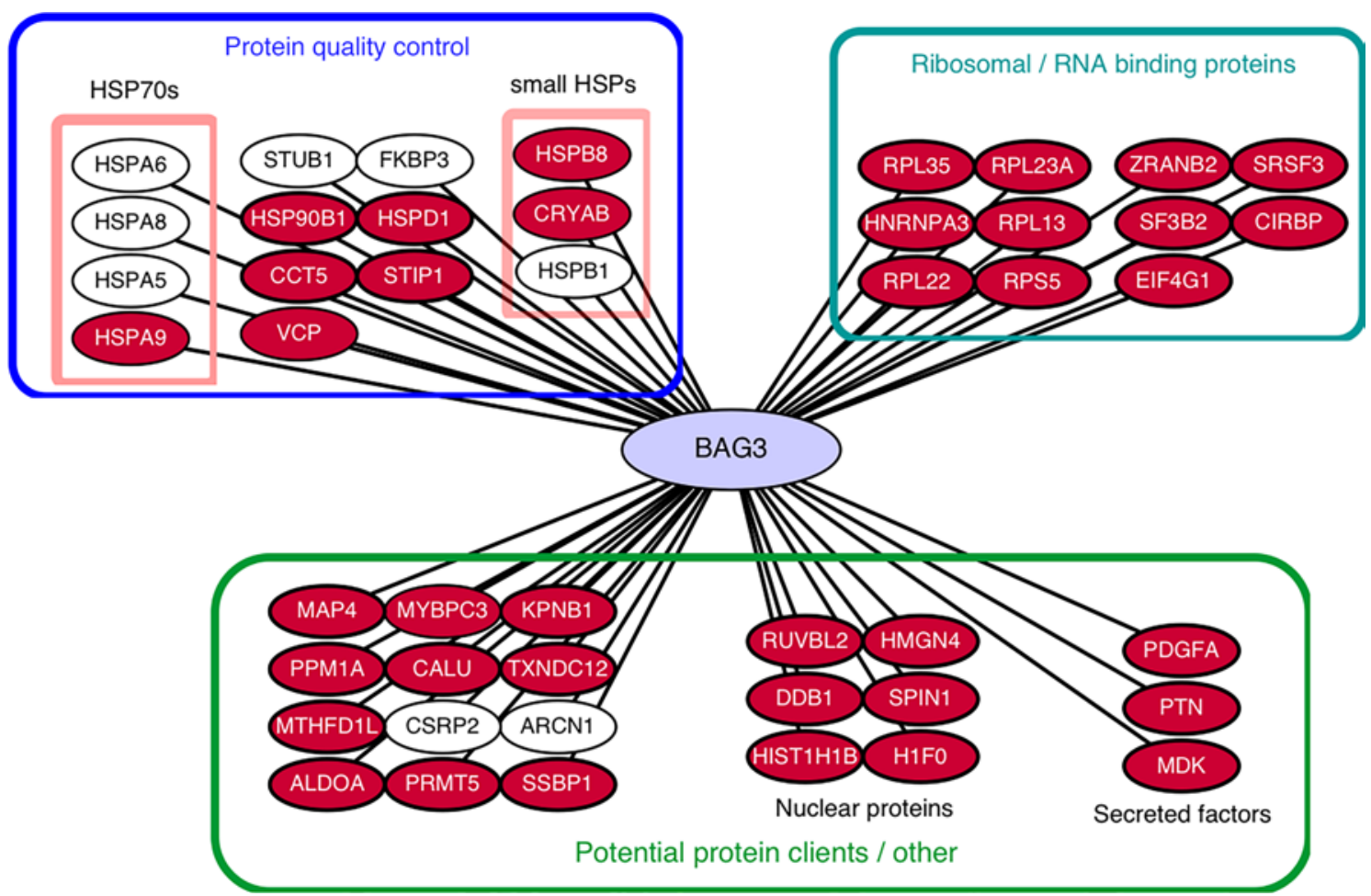

Figure 6. Network representing cardiac-specific BAG3 protein interactions. All 46 interactors identified by affinity purification followed by mass spectrometry (AP-MS) in induced pluripotent stem cell-derived cardiomyocytes (iPS-CMs) with endogenous BAC3-3xFLAC tag were manually grouped by functional categories. White circles indicate interactors that were also identified from undifferentiated iPSCs, red were only identified from iPS-CMs.

measuring doxorubicin toxicity by decreases in both contractility and viability, we found no evidence of increased toxicity in BAG3-mutant cardiomyocytes (Figure 4 and Supplemental Figure 9). Finally, for each drug, the $\mathrm{EC}_{50}$ in WT cells was very close to the reported peak plasma concentration after intravenous infusion in patients, confirming that the assay was sensitive at clinically relevant doses $(34,35,40)$.

We hypothesized that the genetic knockout of BAG3 would change the expression of specific BAG3interacting chaperones that participate in a coordinated stress response. We found that genetically knocking out BAG3 led specifically to a loss of HSPB8 protein levels, while other chaperones were unaffected (Figure 5, A-C). Consistent with findings in immortalized cell lines, BAG3 and HSP70 protein levels were significantly induced by treatment with bortezomib (Figure 5 , A and B) $(26,41)$. Similar findings occurred with MG132 and carfilzomib (data not shown). Surprisingly, of the cardiac-enriched small HSPs reported to interact with BAG3 (HSPB5-8), only HSPB6 and HSPB8 were induced by proteasome inhibition in WT cells (Figure 5, A and B). On the other hand, CRYAB (HSPB5) protein accumulated specifically in $B A G 3^{-1}$ cells after bortezomib treatment (Figure 5, A and C).

Our results implicate HSPB8 as a particularly important partner for BAG3 function in cardiomyocytes. Previous studies reported that BAG3 and HSPB8 are required to activate autophagy to degrade proteotoxic peptides $(23,26,30)$. Therefore, we hypothesized that loss of BAG3 expression, combined with proteasome inhibition, would impair the ability of cardiomyocytes to regulate autophagy that controls compensatory degradation of ubiquitinated proteins. To measure autophagy flux, we used a Western blot assay to monitor LC3 protein levels with and without bafilomycin A1, which inhibits autophagic degradation. The rapidly degraded LC3-II was increased 4- to 6-fold with bafilomycin A1 treatment, consistent with active autophagy flux in cardiomyocytes (Figure 5, D and E). As expected, additional treatment with bortezomib dramatically increased LC3-II levels, consistent with a compensatory induction in autophagy (Figure 5, D and E). However, neither heterozygous nor homozygous knockout of BAG3 significantly changed the levels of LC3-II in any condition. Furthermore, the accumulation of ubiquitinated proteins after bortezomib treatment was the same in WT and BAG3-deficient cardiomyocytes (Figure 5D). These findings indicate that BAG3 is not required for the regulation of bulk autophagy flux in response to proteasome inhibition in human cardiomyocytes; however, it does not rule out a role for targeting specific clients to this pathway. 
To identify novel and cardiac-specific components and/or clients of the BAG3 chaperone complex, we performed AP-MS (42). We used genome engineering to knock in a C-terminal $3 \times$ FLAG affinity tag into the endogenous BAG3 locus on both alleles (Supplemental Figure 10). Both N-terminal and C-terminal BAG3-FLAG showed no difference from WT BAG3 when used for in vitro assays with HSP70 to assess nucleotide exchange factor activity and client release (Jason Gestwicki, personal communication). We also treated WT and BAG3-FLAG iPS-CMs with bortezomib and found no difference in contractility after 48 hours of exposure (data not shown). We then performed AP-MS on both iPS-CMs and undifferentiated iPSCs. In both cell types we also performed AP-MS on cells lacking a $3 \times$ FLAG tag in order to exclude nonspecific interactions. We identified a total of 46 high-confidence interactions with the endogenous BAG3-FLAG in iPS-CMs (Figure 6 and Supplemental Table 1). We found that chaperones and related proteins comprised the single largest group of interactors that we identified, supporting our hypothesis that BAG3 regulates protein quality control pathways in the heart. We did not observe any proteins directly associated with autophagy in our interaction network, which is consistent with the lack of effect of BAG3 in regulating LC3 flux in our system. As expected, the primary overlap between interactors identified from undifferentiated iPSCs and iPS-CMs occurred among ubiquitously expressed chaperones and their associated proteins. However, additional chaperone-related proteins were identified only from cardiomyocytes with the endogenous BAG3 tag, including HSPB8 and CRYAB. We were interested to find that other small HSPs expressed in cardiomyocytes were not present, including HSPB6 and HSPB7. Interestingly, several ribosomal and other RNA-binding proteins were also identified as part of the cardiac-specific BAG3 complex. Although unpredicted, these results support a recently described role for the BAG3-HSP70-HSPB8 complex in processing stress granules and defective ribosomal products, which may represent an underappreciated aspect of protein quality control (43). The remaining identified interactors were a variety of cytoplasmic, sarcomeric, nuclear, and secreted proteins that could be targets of the BAG3 chaperone complex, or they suggest a link to other cellular functions.

\section{Discussion}

Our study shows that disease phenotypes consistent with dilated cardiomyopathy can be recapitulated by de novo engineering a series of isogenic BAG3 mutations in an iPSC line from a healthy donor. Previous iPSC studies of genetic cardiomyopathy and drug toxicity have taken an opposite approach that relies on patientderived samples, with some using genome engineering to correct mutations to create an isogenic control (4446). Although powerful, this approach often requires the comparison of cell lines in a mutational series with different genetic backgrounds. Our approach, however, allows the direct comparison of different mutations in a single isogenic background, which will be crucial for further dissecting mechanisms that correlate genotype and phenotype, as seen with BAG3. Indeed, missense mutations in conserved domains of the BAG3 protein cause different, although severe, clinical phenotypes $(4,11)$. In future studies we hope to characterize the functional effects of missense mutations in key protein-protein interaction domains. For simplicity, we first modeled the genetic loss of BAG3 function given that a large proportion of cardiomyopathy-associated mutations are nonsense, frameshift, or deletions $(8,9)$. We hypothesized that the pathology in these patients is mediated by haploinsufficiency of the BAG3 gene, although it has not been formally demonstrated that these mutations are associated with reduced levels of BAG3 mRNA or protein in human heart. Importantly, we discovered that heterozygous BAG3 mutations led to an approximately 50\% reduction in the BAG3 protein and produced a phenotype in human cardiomyocytes that recapitulates human disease. Surprisingly, in several assays, both partial and complete loss of BAG3 function similarly compromised cardiomyocyte physiology. This highlights the importance of strictly regulating BAG3 expression levels, further supported by the observation that several stimuli that lead to cellular stress induce BAG3 expression (47). This effect likely explains why BAG3 mutations have been associated with both inherited and sporadic cardiomyopathy, the latter of which may result from mutations that subtly affect BAG3 function or expression and manifest as a failure to compensate for an acquired stress. These factors could include infection, ischemia, medications, or exposure to other toxins. Conversely, manipulating the pathways regulated by BAG3 may also protect against cardiac injury. A recent study supporting this hypothesis reported that exogenous BAG3 could protect against cardiac injury from hypoxia-ischemia in mice (48).

We studied the effect of proteasome inhibitors on BAG3-mutant cardiomyocytes for several reasons. First, proteasome inhibitors are useful probes to induce proteotoxic stress, because they inhibit degradation of ubiquitinated proteins, leading to accumulation of potentially aggregation-prone and toxic peptides. Second, 
proteasome inhibition induces BAG3 and related chaperones, which may facilitate alternative degradation pathways $(26,41)$. There is limited evidence that BAG3 may itself directly regulate the proteasome (49). Finally, in a clinical setting, proteasome inhibitors cause cardiac toxicity in an unpredictable subset of patients $(31,50)$. We demonstrate that even partial loss of BAG3 function in human cardiomyocytes leads to severe decompensation in contractile function and myofilament integrity after a single exposure to bortezomib or carfilzomib, confirming that, importantly, BAG3 co-chaperone function protects against proteotoxic stress. This finding has potential clinical implications, because patients with genetic variants in BAG3 or functionally related genes could be predisposed to severe cardiotoxicity from this class of medication. In addition, human iPS-CM models such as ours could be useful for developing safer versions of these and other drugs.

Our data support a model in which BAG3 maintains myofibril function and protects the heart from injury by interacting with multiple families of chaperone proteins. Rigorous biochemical studies demonstrate that BAG3 acts as a co-chaperone by linking the HSP70 and HSPB families and modulating their function $(18,22)$, while cellular experiments support the activity of a BAG3-HSP70-HSPB ternary complex $(23,43)$. Using AP-MS, we identified specific proteins in the HSP70 and small HSP families that interact with BAG3 in cardiomyocytes, which are also coregulated by proteotoxic stress. For example, our data indicate that CRYAB and HSPB8 are the most relevant small HSPs interacting with BAG3 in cardiomyocytes. We further demonstrated that an endogenous epitope tag allowed us to study protein interactions at physiologic levels of expression in the cell type of interest. These data highlight the tissue-specific nature of its role in cardiac biology. With this approach, we identified several potentially novel BAG3-interacting proteins. These include valosin-containing protein (VCP), mutations in which cause inclusion body myopathy, and MYBPC3, which is commonly mutated in hypertrophic and dilated cardiomyopathy $(39,51)$. Interestingly, MYBPC3 was the only sarcomeric protein that was copurified with the BAG3 complex, suggesting that it could be an important client protein. Various proteins identified likely represent additional clients of the BAG3 chaperone complex, while others suggest additional functions for BAG3 in regulating the proteome, including at the level of translation by the ribosome. Future genetic and functional studies of these pathways may reveal mechanisms of heart failure, genetic predisposition to drug toxicity, and novel therapeutic strategies.

\section{Methods}

Human iPSC culture. Control human iPSCs were generated from dermal fibroblasts from a healthy male subject (WTc) using the episomal reprogramming method (52). The subject has a normal ECG and no evidence of cardiac disease with 8 years follow-up. Whole-genome and -exome sequences were obtained for WTc and are available on our laboratory website (https://labs.gladstone.org/conklin/pages/genomic-sequence-data-and-rna-sequence-ips-cells). Exons 1-4 of the BAG3 gene were also amplified by PCR and sequenced by the Sanger method, with no BAG3-coding variants identified. Cells were maintained in mTeSR1 (STEMCELL Technologies) or E8 (Thermo Fisher Scientific) media on growth-factor-reduced Matrigel ( $8 \mu \mathrm{g} / \mathrm{ml}$, BD Biosciences) and passaged every 3-4 days using Accutase (STEMCELL Technologies). ROCK inhibitor Y-27632 (10 $\mathrm{MM}$, Selleckchem) was added to the media for 24 hours after each passage. Cells were cryopreserved as a suspension in fetal bovine serum (FBS, Hyclone) containing 10\% DMSO and $10 \mu \mathrm{M}$ ROCK inhibitor. Cells were used between passage 25-80 for all experiments. Cultures were monitored for mycoplasma on a quarterly basis using a commercial kit (Stratagene).

TALEN and CRISPR construct design. ZiFiT (http://zifit.partners.org/ZiFiT/ChoiceMenu.aspx) was used to design BAG3 and MYBPC3 TALENs. TALENs were constructed using the Voytas laboratory Golden Gate assembly system and backbone vector MR015, as previously described (27). All TALENs used the NN repeat variable di-residues (RVDs). Target sequences for BAG3 TALENs were left TALEN 5'-CCTCTGCCAATGGCCC-3' and right TALEN 5'-AGCAGGCGGCAGCCT-3'. Target sequences for MYBPC3 TALENs were left TALEN 5'-TGACGTCTCTCAGGATGC-3' and right TALEN 5'-TCCTAAAGCTACCTGGC-3'. For designing CRISPR guide RNA targeting BAG3, we used the Zhang lab website (crispr.mit.edu). The guide sequence was cloned into a guide RNA (gRNA) expression vector using the protocol described by the Zhang lab CRISPR resources on Addgene (www.addgene.org/crispr/zhang) (53). pX330-U6-Chimeric_BB_CBh-hSpCas9 (Addgene plasmid 42230) was a gift from Feng Zhang (Broad Institute, Cambridge, Massachusetts, USA). A modified version in which the Cas9 expression cassette was removed ( $\mathrm{pEN102)}$ was a gift from Elphege Nora and Benoit Bruneau (Gladstone Institutes). The sequence of gRNA targeting exon 2 for generating KO2 was 5'-ACCTGTCCACACTGTTTATC-3'. The sequence 
of gRNA targeting exon 4 for generating C-terminal FLAG was 5'-GCAGAGGCTACGGTGCTGCT-3'.

Transfection of iPSCS for gene targeting. Cells were passaged at near-confluent density and $1 \times 10^{6}$ to $2 \times$ $10^{6}$ cells were transfected using the Amaxa Nucleofector $2 \mathrm{~b}$ system and Human Stem Cell Solution I, executing program A-023 (Lonza). For TALEN mutagenesis, we transfected WTc with left and right TALEN plasmids and donor plasmid. For FLAG tagging, we transfected WTc with pX330 and donor plasmid. For BAG3 KO2 we transfected the transgenic CRISPRn line (derived from WTc)(54) with pEN102. In the latter case, doxycycline $(1 \mu \mathrm{g} / \mathrm{ml})$ was added to the media 24 hours before transfection and for 48 hours after to transiently induce Cas9 expression. After transfection, cells were immediately plated onto Matrigelcoated plates and cultured for 24 hours with media containing ROCK inhibitor.

Isolation of modified iPSC clones. Cells transfected with TALEN or Cas9 plasmids along with BAG3knockout vector, MYBPC3-knockout vector, or FLAG-tag vector were cultured in selective media containing $0.5 \mu \mathrm{g} / \mathrm{ml}$ puromycin and $10 \mu \mathrm{g} / \mathrm{ml}$ ROCK inhibitor, starting 48 hours after transfection. Selective media were exchanged daily until stable mCherry-positive colonies remained (5-7 days). After polyclonal enrichment and confirmation of genotype, clonal populations were derived with 1-2 rounds of manual clone picking. For the FLAG-tag line, the pooled population was nucleofected with a plasmid expressing Cre recombinase, and mCherry-negative clones were selected. For the CRISPR BAG3 KO2 lines, clones were picked directly without enrichment. For subcloning, $2 \times 10^{4}$ cells were plated on Matrigel-coated $10-\mathrm{cm}$ tissue culture dishes. After 4-6 days, individual colonies were manually picked and expanded in culture, then divided into one portion for cryopreservation and another portion for genomic DNA extraction (Qiagen DNAeasy Blood \& Tissue kit) and genotyping. Several genotyping strategies were used depending upon the mutation. To genotype clones with integration of BAG3-KO, MYBPC3-KO, or FLAG tag we used PCR assays to distinguish the endogenous and modified alleles. For each predicted integration event we designed primers to amplify across the integration junction at both the $5^{\prime}$ and $3^{\prime}$ end of the targeting vector, as well as a separate primer pair to amplify the unmodified allele. Sequences for all primers used for genotyping are shown in Supplemental Table 1. For selected clones, we sequenced each PCR product to confirm integrity of the integration junctions and to rule out mutations in the untargeted alleles. For offtarget analysis of TALEN-mediated mutant clones we used the TALENoffer software (55). We chose the top 10 off-target loci as predicted by TALENoffer and designed flanking primers to amplify each region by PCR. The PCR products from all 10 loci for $B A G 3^{+/-}$and $B A G 3^{-1-} \mathrm{KO} 1$ lines were sequenced and did not demonstrate any TALEN-induced mutations. To genotype clones with Cas9-mediated frameshift mutations (KO2), we PCR amplified exon 2 and sequenced the products to identify clones with small insertions or deletions. We verified the precise sequence of individual mutant alleles by cloning the PCR products into pCR2.1 (TOPO TA cloning kit, Thermo Fisher Scientific) and sequencing multiple plasmid clones with M13 forward and reverse primers. We performed a droplet digital PCR assay to determine the copy number of FLAG and puromycin insertions (see Supplemental Table 1) as compared to a reference gene RPP30 (Bio-Rad).

Karyotyping. All karyotyping was performed by Cell Line Genetics.

Pluripotency staining for iPSCs. Performed as described previously (27).

iPS-CM differentiation and culture. We used modifications of the WNT modulation method for directed differentiation of iPSCs into cardiomyocytes (56). Freshly passaged iPSCs were seeded on Matrigel-coated 12 -well plates, with initial seeding density optimized for each line $\left(6.25 \times 10^{3}\right.$ to $\left.2.5 \times 10^{4} \mathrm{cells} / \mathrm{cm}^{2}\right)$. Approximately 72 hours after plating (day 0), media were changed to RPMI 1640 with B27 supplement (without insulin) containing CHIR99021 (Tocris). The optimal concentration and exposure time for CHIR was optimized for each line, either $12 \mu \mathrm{M}$ for 24 hours or $6 \mu \mathrm{M}$ for 48 hours. For high-dose CHIR concentrations, the media were changed to RPMI/B27 (without insulin) on day 1, and then RPMI/B27 (without insulin) containing $5 \mu \mathrm{M}$ IWP2 (Tocris) on day 3. For low-dose CHIR concentrations, media were changed to RPMI/B27 (without insulin) containing $5 \mu \mathrm{M}$ IWP2 on day 2. For both protocols, the media containing IWP2 were left for 48 hours and then changed to RPMI/B27 (without insulin) only. After another 48 hours, media were changed to RPMI/B27 containing insulin. Fresh RPMI/B27 was exchanged every 3-4 days thereafter. For purification of iPS-CMs, we used a metabolic selection protocol with glucose-free DMEM containing lactate (57). Cells were replated on day 15-18 of differentiation, and then on day 20-22 media were replaced with DMEM (without glucose, with sodium pyruvate, Thermo Fisher Scientific) supplemented with Glutamax, non-essential amino acids, and buffered lactate (4 mM). Stock-buffered lactate solution was prepared by dissolving sodium L-lactate powder (Sigma-Aldrich) at $1 \mathrm{M}$ concentration in 1 
M HEPES solution. Lactate media were exchanged 2-3 times, with a total exposure of 48 hours for each treatment. After the final lactate treatment (day 24-28), media were changed to RPMI/B27, which was exchanged every 3-4 days thereafter. After day 30, iPS-CMs (hereafter referred to as day $>30$ iPS-CMs) were either replated directly for experiments or cryopreserved. For any given experiment using multiple cell lines, all samples were either directly replated in parallel at the same differentiation stage, or thawed and then replated in parallel.

Immunofluorescent staining of iPS-CMs. Glass coverslips were placed in 12-well plates and coated with Matrigel. Lactate-purified iPS-CMs were prepared as described above and plated at a density of $2 \times 10^{4}$ cells/coverslip. Cells were maintained in culture for 7-14 days and then fixed in 4\% paraformaldehyde for 15 minutes at room temperature. Fixed coverslips were washed repeatedly with PBS containing $0.1 \%$ Triton X-100 (PBS-T), then blocked in a solution of 5\% bovine serum albumin (BSA, Sigma-Aldrich) in PBS-T at room temperature for 1 hour. Primary antibodies were diluted in 5\% BSA solution and incubated overnight at $4^{\circ} \mathrm{C}$. Coverslips were washed again repeatedly with PBS-T and then incubated with secondary antibodies diluted in 5\% BSA solution for 1-2 hours at room temperature. Coverslips were washed a final time and then mounted onto glass slides with VECTASHIELD HardSet with DAPI (Vector Laboratories). Primary antibodies included mouse monoclonal anti-sarcomeric actinin (clone EA-53, Sigma-Aldrich) and rabbit anti-BAG3 (Protein Tech, 10599-1-AP). Secondary antibodies included Alexa Fluor 488 goat anti-mouse IgG, Alexa Fluor 594 goat anti-mouse IgG, Alexa Fluor 488 goat anti-rabbit IgG, and Alexa Fluor 594 goat anti-rabbit IgG (Molecular Probes). All images were taken on a BZ-9000 microscope (Keyence).

Flow cytometry. Flow cytometry was performed as previously described (54) using rabbit anti-BAG3 antibody (Protein Tech, 10599-1-AP) and mouse monoclonal antibody against cardiac troponin-T (clone 13-11, Thermo Fisher Scientific).

Sarcomere scoring in fixed iPS-CMs. Day $>30$ iPS-CMs were cultured on glass coverslips for 10-14 days (initial experiments were performed with a 14-day interval; later experiments with a 10-day interval gave equivalent results). Coverslips were then fixed in $4 \%$ paraformaldehyde, stained with anti- $\alpha$-actinin antibody, and mounted on slides as described above. The slides were then labeled with an alphanumeric code to blind scientists performing the experiment to the identity of each sample. Between 20 and 24 images were taken with a $\times 40$ objective for each slide, with a total of 50-150 cells imaged from each slide. Each cell was then assigned a score between 1 and 5 corresponding to the relative degree of disordered myofilaments as follows: 1, all myofilaments were continuous and well ordered with the majority in parallel; 2, all myofilaments were continuous and well ordered but not in parallel; 3, significantly disordered myofilaments with fragmentation, disintegration of Z-disk structures, or actinin aggregates making up less than $50 \%$ of the total area; 4, significantly disordered myofilaments with fragmentation, disintegration of Z-disk structures, or actinin aggregates making up 50\% or more of the total area; 5 , positive actinin staining, but no identifiable myofilament structure. After all images were scored, the code was broken and each sample assigned to the corresponding cell line. The percentage of cells in each category was calculated for each slide, and the mean and standard deviation was calculated for replicate samples from each cell line. For analysis of KO1 lines, cells from 3 separate differentiation batches were used to seed replicate cultures (WT, $n=8 ; B A G 3^{+/-}$, $\left.n=7 ; B A G 3^{-/-}, n=9\right)$. For KO2 lines, cells from 2 separate differentiation batches were used to seed replicate cultures (WT, $n=7 ; B A G 3^{+/-}, n=4 ; B A G 3^{-/-}, n=8$ ).

Analysis of micropatterned iPS-CMs. The complete methodology has been previously described (58). In brief, day >30 iPS-CMs from BAG3 KO1 lines were thawed on fibronectin-coated wells and recovered for 5 days before seeding on micropatterned polyacrylamide substrates. Singularized hiPSC-CMs $\left(1 \times 10^{5}\right)$ in RPMI 1640 medium plus B27 supplement $(50 \times)$, penicillin $(25 \mu \mathrm{g} / \mathrm{ml})$, and streptomycin $(50 \mu \mathrm{g} / \mathrm{ml})$ (all from Life Technologies) with $5 \mu \mathrm{M}$ Y27623 ROCK inhibitor were seeded and cultured on the top of hydrogel devices. Cell culture medium without ROCK inhibitor was changed after 2 days of culture. After 3 days, 20-30 fps videos of beating single cells and of the movement of microbeads embedded in the hydrogel substrate underneath each cell were acquired in a Zeiss Axiovert $200 \mathrm{M}$ with a Zeiss Axiocam MRm camera, using a $\times 40$ objective. Power contractile output of single hiPSC-CMs was calculated by multiplying the force they generate by the velocity of microbeads during contractions. Cell contractile force was calculated by submitting frames within videos of fluorescent microbeads to algorithms of cross-correlation and traction force microscopy developed in MATLAB. Cross-correlation also generated information on the displacement of each frame and time, which was used to calculate microbead velocity. Cells from 2 
independent differentiation batches were seeded on 3 separate micropatterned devices. Data were analyzed from all cells on devices with $10-\mathrm{kPa}$ substrate (WT, $n=51 ; B A G 3^{+/-}, n=40 ; B A G 3^{-/-}, n=42$ ) as well as $35-\mathrm{kPa}$ substrate (WT, $n=40 ; B A G 3^{+/-}, n=11 ; B A G 3^{-/-}, n=47$ ).

Micropatterned hiPSC-CMs were transfected with RFP-LifeAct (Ibidi) to label sarcomeres and sarcomere length was calculated from the distance between Z-lines in beating cells. For this purpose, we acquired videos of fluorescently labeled micropatterned beating cells. Sarcomere shortening of each cell resulted from subtracting the average sarcomere length of the cell in its contracted state from the average sarcomere length of the cell in its relaxed state. For analysis of sarcomere shortening, measurements from cells seeded on $10-\mathrm{kPa}$ substrate from 2 independent differentiation batches were combined (WT, $n=26 ; B A G 3^{+/-}, n=$ 9; $\left.B A G 3^{-1-}, n=17\right)$. Measurements from cells seeded on $35-\mathrm{kPa}$ substrate were from a single differentiation batch (WT, $n=8 ; B A G 3^{-/}, n=7$ ).

Live sarcomere labeling and scoring in iPS-CMs. Day $>30$ iPS-CMs were dissociated and counted as described above. Then $0.5 \times 10^{6}$ to $1.5 \times 10^{6}$ cells were resuspended in $100 \mu 1$ Human Stem Cell Solution I with $2 \mu \mathrm{g}$ of $\alpha$-actinin-mKate 2 reporter plasmid and transfected with the Amaxa Nucleofector $2 \mathrm{~b}$ system, executing program G-009 (Lonza). After transfection, cells were immediately plated onto Matrigel-coated 48-well plates and cultured for 24 hours with media containing ROCK inhibitor. The cells recovered in culture for 7 days before starting experiments. Imaging was performed on a Zeiss Axio Observer microscope with an automated stage. On day 0 of the experiment, 5-8 individual $\times 40$ fields of interest containing cells with well-labeled sarcomeres were acquired from each well, and the $\mathrm{X} / \mathrm{Y} / \mathrm{Z}$ coordinates were saved for each field. Fresh media containing either $0.01 \%$ DMSO or $0.1 \mu \mathrm{M}$ bortezomib were added to each well, with 4 replicate cultures per cell line treated with each condition. The saved coordinates were imaged again at 24 and 48 hours after addition of drug. After all images were acquired, each cell was assigned a score for degree of myofilament disarray as described above. The percentage of cells in each category was calculated for replicate cultures.

Drug treatment and contractility assays in iPS-CMs. Day $>30$ iPS-CMs were dissociated and counted as described above. Cells were plated in 48-well or 96-well plates at a density of $4 \times 10^{4}$ or $2 \times 10^{4}$ cells/ well respectively. Cells were maintained in culture for 7-10 days before beginning experiments. Bortezomib (Cell Signaling Technology), carfilizomib (Selleckchem), and doxorubicin (Cell Signaling Technology) were dissolved in DMSO at $10 \mathrm{mM}$ concentration, filtered through a $0.22-\mu \mathrm{m}$ syringe filter, and stored in aliquots at $-20^{\circ} \mathrm{C}$. Contractile motion was captured by automated video microscopy and analyzed using the Cellogy Pulse system. Fresh media were exchanged, and the cells equilibrated in the incubator for 2-4 hours before obtaining baseline data. Drugs or vehicle were diluted to a $3 \times$ final concentration in complete media and added to the existing media in each well for the corresponding final concentration. Data were acquired on the Cellogy Pulse system every 24 hours, and the basal media were exchanged 48 hours after addition of drugs and again every 48 hours after. The contractility index was calculated by dividing the contraction peak height at each time point from the baseline value for each well. The normalized beat rate was calculated in a similar fashion.

For testing adrenergic agonists, $10 \mathrm{mM}$ norepinephrine bitartrate (Sigma-Aldrich) was prepared in water with $100 \mathrm{mM}$ citric acid, and $10 \mathrm{mM}$ phenylephrine hydrochloride (Sigma-Aldrich) and $10 \mathrm{mM}$ isoproterenol hydrochloride (Sigma-Aldrich) were prepared in water. All solutions were filtered through a $0.22-\mu \mathrm{m}$ syringe filter. Norepinephrine and phenylephrine were aliquoted and stored at $-20^{\circ} \mathrm{C}$, and isoproterenol was prepared fresh. Baseline data were obtained as described and then again 1 hour after addition of drug. For repetitive norepinephrine treatment, fresh media were exchanged each morning, with baseline data obtained 4 hours later. Additional media containing $3 \times$ norepinephrine or vehicle was then added, with data acquisition 1 hour later. This process was repeated for 5 consecutive days.

Resazurin viability assay in iPS-CMs. Cells used for contractility assays recovered for 5 days after removal of drug. Then, 7 days from the beginning of the experiment, media were replaced with fresh RPMI/B27 with $10 \%$ PrestoBlue reagent (Thermo Fisher Scientific) and incubated for 1 hour at $37^{\circ} \mathrm{C}$. Fluorescence intensity was measured with $560 \mathrm{~nm}$ excitation and $590 \mathrm{~nm}$ emission on a SpectraMax i3 plate reader (Molecular Devices). Background measurements from wells with media only were subtracted from all samples. For each cell line, the fluorescence intensity was normalized to control wells with no drug added.

Western blot analysis. Cells were dissociated with accutase (iPSCs) or $0.25 \%$ trypsin/EDTA (iPS$\mathrm{CMs}$ ), and cell pellets were washed once with PBS. Cells were lysed in RIPA buffer containing complete Protease Inhibitors (Roche), and the protein concentration was measured using a Bradford-based assay 
(Bio-Rad). Samples were prepared at equal concentration in NuPaGE LDS sample buffer, loaded on NuPAGE 4\%-12\% Bis-Tris gels, and run in NuPAGE MES running buffer (Thermo Fisher Scientific). Proteins were transferred to nitrocellulose membranes using the iBlot transfer system (Thermo Fisher Scientific). Membranes were blocked in Odyssey blocking buffer (LiCOR) for 1 hour at room temperature. Primary antibodies were diluted in blocking buffer with $0.1 \%$ Tween 20 . Membranes were incubated in primary antibody solution at $4^{\circ} \mathrm{C}$ overnight, then washed with 3 aliquots of PBS with $0.1 \%$ Tween 20 (PBS-T). Fluorescently labeled secondary antibodies were diluted in blocking buffer with $0.1 \%$ Tween 20 and $0.01 \%$ SDS. Membranes were incubated in secondary antibody solution for 1 hour at room temperature, then washed with 3 aliquots of PBS-T. After the final washing step, membranes were imaged immediately with an Odyssey Fc fluorescent imaging system (LI-COR). Individual band intensities were measured using Image Studio software (LI-COR), and the intensity of each band of interest was normalized to the loading control (GAPDH) for the corresponding sample. Primary antibodies used included mouse anti-HSP70/HSP72 (clone C92F3A-5, Enzo Life Sciences), rabbit anti-BAG3 (Protein Tech, 10599-1-AP), mouse anti- $\alpha$ B crystallin (clone 1B6.1-3G4, Enzo Life Sciences), rabbit anti-Hsp20 (clone HSPB6, Abcam, ab13492), mouse anti-cvHSP (clone HSPB7, Abcam, ab57093), rabbit anti-Hsp22 (clone HSPB8, Abcam, ab96837), mouse anti-FLAG (Sigma-Aldrich, F3165), and rabbit anti-GAPDH (Abcam, ab9485). Secondary antibodies used included IRDye 680LT donkey anti-mouse and IRDye $800 \mathrm{CW}$ donkey anti-rabbit (LI-COR).

Affinity purification of BAG3 interacting partners. For AP-MS, 2 replicates each of BAG33xFLAG/3xFLAG undifferentiated iPSCs and differentiated iPS-CMs were used. Two replicates of WTc iPSCs and iPS-CMs (not expressing BAG3-3xFLAG) were used as controls for nonspecific interactions. For iPSC pulldowns, 4 $\times 15 \mathrm{~cm}$ dishes at approximately $80 \%$ confluence were used. For iPS-CM pulldowns, $25 \times 10^{6}$ to $30 \times 10^{6}$ lacatate-purified cells at day 30 of differentiation were used. Cells were harvested in PBS by scraping and resuspended in 2 volumes of lysis buffer $(0.1 \% \mathrm{NP}-40,300 \mathrm{mM} \mathrm{NaCl}, 25 \%$ glycerol, $2 \mathrm{mM} \mathrm{MgCl}, 0.5 \mathrm{mM}$ EDTA, 0.5 mM EGTA, $1 \mathrm{mM}$ PMSF, and $1 \mathrm{mM}$ DDT in $50 \mathrm{mM}$ HEPES-NaOH pH 8.0), supplemented with cOmplete Protease Inhibitor Cocktail (Roche) and Benzonase Nuclease (50 U/ml, Sigma-Aldrich). Cells were lysed by 4 freeze-thaw cycles, followed by incubation for 20 minutes at $4^{\circ} \mathrm{C}$. After removing the insoluble fraction, protein extracts were diluted 3-fold to reduce salt content and incubated with $30 \mu 1$ antiFLAG M2 Magnetic Beads slurry (Sigma-Aldrich) for 2 hours. Beads were rinsed in wash buffer (3 washes in $0.01 \%$ NP-40, 1 mM PMSF, 0.5\% EDTA, 0.5\% EGTA in $50 \mathrm{mM}$ HEPES, pH 8.0; 1 wash in buffer without NP-40). FLAG-enriched proteins were reduced (5 mM TCEP), alkylated (15 mM iodoacetamide), and digested with $1 \%(\mathrm{w} / \mathrm{v})$ trypsin overnight. Resulting peptides were desalted by UltraMicroSpin columns (The Nest Group) and dried.

Peptides were resuspended in $0.1 \%$ formic acid and analyzed by liquid chromatography-tandem mass spectrometry (LC-MS/MS) with an Easy-nLC 1000 (Thermo Fisher Scientific) coupled to an Orbitrap Fusion Tribrid Mass Spectrometer (Thermo Fisher Scientific). Online LC separation was carried out using a $75 \mu \mathrm{m} \times 25 \mathrm{~cm}$ fused silica IntregraFrit capillary column (New Objective) packed in-house with 1.9- $\mathrm{mm}$ Reprosil-Pur C18 AQ reverse-phase resin (Dr. Maisch-GmbH). Peptides were eluted at a flow rate of 300 $\mathrm{nl} /$ min using a linear gradient of 5\%-30\% B for 45 minutes, and 30\%-95\% B for 25 minutes (mobile phase buffer A: $100 \% \mathrm{H}_{2} \mathrm{O} / 0.1 \%$ formic acid; mobile phase buffer B: 100\% ACN/0.1\% formic acid). Survey scans of peptide precursors from 400 to $1,600 \mathrm{~m} / z$ were performed at $120 \mathrm{~K}$ resolution in the Orbitrap, with an AGC target of $2 \times 10^{5}$, and a maximum injection time of $100 \mathrm{~ms}$. MS/MS was performed by isolation with the quadrupole, HCD fragmentation with normalized collision energy of $30 \%$, and rapid scan MS analysis in the ion trap. The MS/MS ion count target was set to $10^{4}$ and the max injection time was 35 $\mathrm{ms}$. Precursors with charge state 2-7 were sampled for MS/MS and dynamically excluded for 20 seconds (tolerance of $10 \mathrm{ppm}$ ). Monoisotopic precursor selection was turned on, and the instrument was run in top speed mode with 3-second cycles.

For protein identification and quantification, MaxQuant software v1.5.3.30 was used (59). MS/MS spectra were searched against the November 2016 release of the UniProt complete human proteome sequence database, modified to include the FLAG peptide sequence. MaxQuant was run on default parameters, allowing for 2 maximum missed cleavages, with a first search peptide tolerance of $20 \mathrm{ppm}$ and a main search peptide tolerance of $4.5 \mathrm{ppm}$. Methionine oxidation and $\mathrm{N}$-terminal acetylation were set as variable modifications, and carbamidomethylation of cysteines as fixed modification. The 'match between runs' setting was activated (window of 7 minutes) to improve peptide identification. 
For the analysis of data, proteins with 2 or fewer peptides identified were discarded, as were typical common contaminant proteins (downloaded from http://maxquant.org/). Since the 2 sets were run separately, protein intensity ratios (BAG3 ${ }^{3 \times F L A G / 3 x F L A G} / \mathrm{WTC}$ ) were calculated separately for each cell type and for every replicate. Intensity values for missing proteins in each sample were inferred as $10 \%$ of the lowest intensity value for that sample. $Z$ scores were calculated and $P$ values were corrected for multiple testing using the Bejamini-Hockberg procedure (60). We selected as interactors those proteins with a significant $P$ value $(P<0.05)$ across the replicates. A list of significant hits can be found in Supplemental Table 1).

Software and statistical analysis. Data analysis and graphing was performed with GraphPad Prism v6.0h software. Statistical tests were performed in Prism using an unpaired 2-tailed $t$ test with equal variance, or ANOVA with Bonferroni's test for multiple comparisons. A $P$ value less than 0.05 was considered significant. $\mathrm{EC}_{50}$ and $\mathrm{LD}_{50}$ values with $95 \%$ confidence intervals were calculated from dose-response curves in Prism using $\log$ (inhibitor) versus normalized response with variable slope. Analysis of BAG3-interacting partners was done using R (61) v3.3.0, and the visualization figure was created using Cytoscape v3.4.0 (62). Generation of Venn diagrams was done using BioVenn (http://www.cmbi.ru.nl/cdd/biovenn/index.php) (63).

Study approval. Derivation and use of human iPSCs was approved by the UCSF Committee on Human Research, San Francisco, CA (study number 10-02521). All subjects provided informed consent prior to participation.

\section{Author contributions}

LMJ and BRC conceived the project. LMJ designed the majority of experiments and performed them with assistance from AT, CLJ, and JCY. AT and JAPB generated the endogenous BAG3-3xFLAG iPSC line. JAPB performed AP-MS analysis with assistance from RMK. AJSR generated the micropatterned devices and performed all associated assays. JCY and MAM generated the $M Y B P C 3^{+/-}$iPSC line. NH assisted with design and construction of the $\alpha$-actinin fluorescent reporter plasmid. BLP, DS, NJK, and PLS contributed to valuable conceptual discussions and experimental planning. LMJ and BRC composed the manuscript with assistance from all authors.

\section{Acknowledgments}

We thank members of the Conklin laboratory and the Gladstone Stem Cell Core laboratory for technical assistance; Matthew Porteus and Meghdad Rahdar (Stanford University) for providing us with the TALEN backbone plasmid MR015; Elphege Nora and Benoit Bruneau (Gladstone Institutes) for providing us with the CRISPR gRNA expression plasmid pEN102; Jennifer Rauch, Jason Gestwicki (UCSF), and Harm Kampinga (The University Medical Center Groningen, Groningen, The Netherlands) for discussion and advice. L.M.J. was supported by a postdoctoral fellowship from the California Institute of Regenerative Medicine (CIRM; TG2-01160) and a Career Development Award from the National Institute of Child Health and Development (1K12HD072222). J.A.P.B. was supported by a "La Caixa" International Fellowship and an American Heart Association predoctoral fellowship award (13PRE16120013). B.L.P. was supported by NIH 1R21HL13099301. A.J.S.R. was supported by an American Heart Association postdoctoral fellowship (14POST18360018). A.T. and C.L.J. were supported by a training grant from CIRM (TB1-01188). M.A.M. was supported by a postdoctoral fellowship from the Canadian Institutes of Health Research (number 129844). N.H. was supported by postdoctoral fellowships from CIRM (TG2-01160) and the National Institute of Heart, Lung, and Blood (T32 HL007544). B.R.C. is supported by the Gladstone Institutes and NIH (U01HL100406, P01HL089707, and R01HL130533). All potential conflicts of interest are examined and managed by the Gladstone Institutes Conflicts of Interest in Research Committee.

Address correspondence to: Bruce R. Conklin, Gladstone Institute of Cardiovascular Disease, 1650 Owens Street, San Francisco, California 94158, USA. Phone: 415.734.2712; Email: bconklin@gladstone.ucsf.edu.

1. Willis MS, Patterson C. Proteotoxicity and cardiac dysfunction--Alzheimer's disease of the heart? N Engl J Med 2013;368(5):455-464.

2. McLendon PM, Robbins J. Proteotoxicity and cardiac dysfunction. Circ Res. 2015;116(11):1863-1882

3. Vicart P, et al. A missense mutation in the alphaB-crystallin chaperone gene causes a desmin-related myopathy. Nat Genet. 1998;20(1):92-95. 
4. Selcen D et al. Mutation in BAG3 causes severe dominant childhood muscular dystrophy. Ann Neurol. 2009;65(1):83-89.

5. Tarone G, Brancaccio M. Keep your heart in shape: molecular chaperone networks for treating heart disease. Cardiovasc Res. 2014;102(3):346-361.

6. Ruparelia AA, Oorschot V, Vaz R, Ramm G, Bryson-Richardson RJ. Zebrafish models of BAG3 myofibrillar myopathy suggest a toxic gain of function leading to BAG3 insufficiency. Acta Neuropathol. 2014;128(6):821-833.

7. Quintana MT, et al. Cardiomyocyte-specific human Bcl2-associated anthanogene 3 P209L expression induces mitochondrial fragmentation, Bcl2-associated anthanogene 3 haploinsufficiency, and activates p38 signaling. Am J Pathol. 2016;186(8):1989-2007.

8. Norton N, et al. Genome-wide studies of copy number variation and exome sequencing identify rare variants in BAG3 as a cause of dilated cardiomyopathy. Am J Hum Genet. 2011;88(3):273-282.

9. Villard E, et al. A genome-wide association study identifies two loci associated with heart failure due to dilated cardiomyopathy. Eur Heart J. 2011;32(9):1065-1076.

10. Chami N, et al. Nonsense mutations in BAG3 are associated with early-onset dilated cardiomyopathy in French Canadians. Can J Cardiol. 2014;30(12):1655-1661.

11. Franaszczyk M, et al. The BAG3 gene variants in Polish patients with dilated cardiomyopathy: four novel mutations and a genotype-phenotype correlation. J Transl Med. 2014;12:192.

12. d'Avenia M, et al. A novel miR-371a-5p-mediated pathway, leading to BAG3 upregulation in cardiomyocytes in response to epinephrine, is lost in Takotsubo cardiomyopathy. Cell Death Dis. 2015;6:e1948.

13. Citro R, et al. Polymorphisms of the antiapoptotic protein bag3 may play a role in the pathogenesis of tako-tsubo cardiomyopathy. Int J Cardiol. 2013;168(2):1663-1665.

14. Feldman AM, et al. Decreased levels of BAG3 in a family with a rare variant and in idiopathic dilated cardiomyopathy. $J$ Cell Physiol. 2014;229(11):1697-1702.

15. Homma S, Iwasaki M, Shelton GD, Engvall E, Reed JC, Takayama S. BAG3 deficiency results in fulminant myopathy and early lethality. Am J Pathol. 2006;169(3):761-773.

16. Youn DY, et al. Bis deficiency results in early lethality with metabolic deterioration and involution of spleen and thymus. Am $J$ Physiol Endocrinol Metab. 2008;295(6):E1349-E1357.

17. Franceschelli S, Rosati A, Lerose R, De Nicola S, Turco MC, Pascale M. Bag3 gene expression is regulated by heat shock factor 1. J Cell Physiol. 2008;215(3):575-577.

18. Rauch JN, Gestwicki JE. Binding of human nucleotide exchange factors to heat shock protein 70 (Hsp70) generates functionally distinct complexes in vitro. J Biol Chem. 2014;289(3):1402-1414

19. Rauch JN, Zuiderweg ER, Gestwicki JE. Non-canonical interactions between heat shock cognate protein 70 (Hsc70) and Bcl2associated anthanogene (BAG) co-chaperones are important for client release. J Biol Chem. 2016;291(38):19848-19857.

20. Fuchs M, et al. Identification of the key structural motifs involved in HspB8/HspB6-Bag3 interaction. Biochem J. 2009;425(1):245-255.

21. Shemetov AA, Gusev NB. Biochemical characterization of small heat shock protein HspB8 (Hsp22)-Bag3 interaction. Arch Biochem Biophys. 2011;513(1):1-9.

22. Rauch JN, et al. BAG3 is a modular, scaffolding protein that physically links heat shock protein 70 (Hsp70) to the small heat shock proteins. J Mol Biol. 2017;429(1):128-141.

23. Carra S, Seguin SJ, Lambert H, Landry J. HspB8 chaperone activity toward poly(Q)-containing proteins depends on its association with Bag3, a stimulator of macroautophagy. J Biol Chem. 2008;283(3):1437-1444.

24. Gamerdinger M, Kaya AM, Wolfrum U, Clement AM, Behl C. BAG3 mediates chaperone-based aggresome-targeting and selective autophagy of misfolded proteins. EMBO Rep. 2011;12(2):149-156.

25. Arndt V, et al. Chaperone-assisted selective autophagy is essential for muscle maintenance. Curr Biol. 2010;20(2):143-148.

26. Minoia M, et al. BAG3 induces the sequestration of proteasomal clients into cytoplasmic puncta: implications for a proteasome-to-autophagy switch. Autophagy. 2014;10(9):1603-1621.

27. Miyaoka Y, et al. Isolation of single-base genome-edited human iPS cells without antibiotic selection. Nat Methods. 2014;11(3):291-293

28. Heidersbach A, et al. microRNA-1 regulates sarcomere formation and suppresses smooth muscle gene expression in the mammalian heart. Elife. 2013;2:e01323.

29. Ribeiro AJ, et al. Contractility of single cardiomyocytes differentiated from pluripotent stem cells depends on physiological shape and substrate stiffness. Proc Natl Acad Sci U S A. 2015;112(41):12705-12710.

30. Rapino F, Jung M, Fulda S. BAG3 induction is required to mitigate proteotoxicity via selective autophagy following inhibition of constitutive protein degradation pathways. Oncogene. 2014;33(13):1713-1724.

31. Nowis D, et al. Cardiotoxicity of the anticancer therapeutic agent bortezomib. Am J Pathol. 2010;176(6):2658-2668.

32. Enrico O, et al. Unexpected cardiotoxicity in haematological bortezomib treated patients. Br J Haematol. 2007;138(3):396-397.

33. Maddah M, et al. A non-invasive platform for functional characterization of stem-cell-derived cardiomyocytes with applications in cardiotoxicity testing. Stem Cell Reports. 2015;4(4):621-631.

34. Kyprolis (carfilzomib) for injection [package insert]. Thousand Oaks, CA: Onyx Pharmaceuticals, Inc; 2012.

35. Velcade (bortezomib) for injection [package insert]. Cambridge, MA: Millennium Pharmaceuticals, Inc; 2005.

36. Huebsch N, et al. Miniaturized iPS-cell-derived cardiac muscles for physiologically relevant drug response analyses. Sci Rep. 2016;6:24726

37. Wyles SP, et al. Modeling structural and functional deficiencies of RBM20 familial dilated cardiomyopathy using human induced pluripotent stem cells. Hum Mol Genet. 2016;25(2):254-265.

38. Sun N, et al. Patient-specific induced pluripotent stem cells as a model for familial dilated cardiomyopathy. Sci Transl Med. 2012;4(130):130ra47.

39. Haas J, et al. Atlas of the clinical genetics of human dilated cardiomyopathy. Eur Heart J. 2015;36(18):1123-135a.

40. Barpe DR, Rosa DD, Froehlich PE. Pharmacokinetic evaluation of doxorubicin plasma levels in normal and overweight patients with breast cancer and simulation of dose adjustment by different indexes of body mass. Eur J Pharm Sci. 2010;41(3-4):458-463.

41. Du ZX, et al. Proteasome inhibitor MG132 induces BAG3 expression through activation of heat shock factor 1. J Cell Physiol. 
2009;218(3):631-637.

42. Kaake RM, Wang X, Huang L. Profiling of protein interaction networks of protein complexes using affinity purification and quantitative mass spectrometry. Mol Cell Proteomics. 2010;9(8):1650-1665.

43. Ganassi M, et al. A surveillance function of the HSPB8-BAG3-HSP70 chaperone complex ensures stress granule integrity and dynamism. Mol Cell. 2016;63(5):796-810.

44. Burridge PW, et al. Human induced pluripotent stem cell-derived cardiomyocytes recapitulate the predilection of breast cancer patients to doxorubicin-induced cardiotoxicity. Nat Med. 2016;22(5):547-556.

45. Liang P, et al. Drug screening using a library of human induced pluripotent stem cell-derived cardiomyocytes reveals diseasespecific patterns of cardiotoxicity. Circulation. 2013;127(16):1677-1691.

46. Wang $\mathrm{G}$, et al. Modeling the mitochondrial cardiomyopathy of Barth syndrome with induced pluripotent stem cell and hearton-chip technologies. Nat Med. 2014;20(6):616-623.

47. Behl C. Breaking BAG: The co-chaperone BAG3 in health and disease. Trends Pharmacol Sci. 2016;37(8):672-688.

48. Su F, et al. Bcl-2-associated athanogene 3 protects the heart from ischemia/reperfusion injury. JCI Insight. 2016;1(19):e90931.

49. Chen Y, et al. Bcl2-associated athanogene 3 interactome analysis reveals a new role in modulating proteasome activity. Mol Cell Proteomics. 2013;12(10):2804-2819.

50. Grandin EW, Ky B, Cornell RF, Carver J, Lenihan DJ. Patterns of cardiac toxicity associated with irreversible proteasome inhibition in the treatment of multiple myeloma. J Card Fail. 2015;21(2):138-144.

51. Meyer H, Weihl CC. The VCP/p97 system at a glance: connecting cellular function to disease pathogenesis. J Cell Sci. 2014;127(Pt 18):3877-3883.

52. Okita K, et al. A more efficient method to generate integration-free human iPS cells. Nat Methods. 2011;8(5):409-412.

53. Cong L, et al. Multiplex genome engineering using CRISPR/Cas systems. Science. 2013;339(6121):819-823.

54. Mandegar MA, et al. CRISPR interference efficiently induces specific and reversible gene silencing in human iPSCs. Cell Stem Cell. 2016;18(4):541-553.

55. Grau J, Boch J, Posch S. TALENoffer: genome-wide TALEN off-target prediction. Bioinformatics. 2013;29(22):2931-2932.

56. Lian X, et al. Directed cardiomyocyte differentiation from human pluripotent stem cells by modulating Wnt/ $\beta$-catenin signaling under fully defined conditions. Nat Protoc. 2013;8(1):162-175.

57. Tohyama S, et al. Distinct metabolic flow enables large-scale purification of mouse and human pluripotent stem cell-derived cardiomyocytes. Cell Stem Cell. 2013;12(1):127-137.

58. Ribeiro AJS, et al. Multi-imaging method to assay the contractile mechanical output of micropatterned human iPSC-derived cardiac myocytes. Circ Res. 2017;120(10):1572-1583.

59. Cox J, Mann M. MaxQuant enables high peptide identification rates, individualized p.p.b.-range mass accuracies and proteomewide protein quantification. Nat Biotechnol. 2008;26(12):1367-1372.

60. Benjamini Y, Hochberg Y. Controlling the false discovery rate: a practical and powerful approach to multiple testing. $J R$ Stat Soc Series B Stat Methodol. 1995;57(1):289-300.

61. R Core Team. R: A language and environment for statistical computing. R Foundation for Statistical Computing, Vienna, Austria. http://www.R-project.org/. Accessed June 15, 2017.

62. Shannon P, et al. Cytoscape: a software environment for integrated models of biomolecular interaction networks. Genome Res. 2003;13(11):2498-2504.

63. Hulsen T, de Vlieg J, Alkema W. BioVenn - a web application for the comparison and visualization of biological lists using areaproportional Venn diagrams. BMC Genomics. 2008;9:488. 